\title{
Two Case Studies on Inferior Mesenteric Artery Embolization for Primary Postpartum Hemorrhage
}

\author{
Department of Interventional Radiology, Japanese Red Cross Medical Center, Japan
}

Naoki Kawakami, Fumiya Uchiyama, Akinori Harada, Tetsuhisa Yamada, Jun-ichi Nishimura

\begin{abstract}
Postpartum hemorrhage (PPH) remains a leading cause of maternal mortality. Transcatheter arterial embolization (TAE) is an effective treatment option for PPH. Among the vessels embolized, the inferior mesenteric artery (IMA) is usually not the first choice for TAE, because it is a rare source of bleeding in PPH. In this report, we describe our experience with two patients with PPH, in whom the IMA was selected on the basis of contrast-enhanced computed tomography (CECT) findings, and prompt hemostasis was achieved with TAE. CECT can provide useful clues as to the culprit artery based on the anatomical location of the hematoma and extravasation. These useful findings of CECT allow prompt TAE of rare sources of bleeding in $\mathrm{PPH}$, such as the IMA.
\end{abstract}

Key words: Contrast-enhanced computed tomography, Inferior mesenteric artery, Postpartum hemorrhage (Interventional Radiology Advance Publication)

\section{Introduction}

Postpartum hemorrhage (PPH) remains a leading cause of maternal mortality despite advances in treatment; it is also responsible for approximately $11 \%$ of maternal deaths worldwide $[1,2]$. Therefore, timely and appropriate multidisciplinary treatment is a priority.

Transcatheter arterial embolization (TAE) is an effective treatment for PPH and is an alternative to hysterectomy [3]. In most patients with $\mathrm{PPH}$, either the uterine arteries or the anterior divisions of the internal iliac arteries are first selected for TAE, even when extravasation is not observed [1]. If TAE of these arteries and other branches of the internal iliac arteries fails to achieve hemostasis, other potential arterial sources are considered. Among these, the inferior mesenteric artery (IMA) is an uncommon bleeding source [4]. Studies on TAE of the IMA as a treatment for PPH are extremely limited [5].

We present two patients with primary $\mathrm{PPH}$ in whom we first performed TAE of the IMA on the basis of contrast- enhanced computed tomography (CECT) findings, and prompt hemostasis was achieved in both patients.

\section{Case Reports}

\section{Case 1}

A 29-year-old woman was transferred to our hospital because of vaginal bleeding persisting for $8 \mathrm{~h}$ after vacuum extraction delivery. On arrival, her vital signs and laboratory data were as follows: blood pressure, $120 / 78 \mathrm{mmHg}$; heart rate, 114 beats/min; shock index, 0.95; hemoglobin level, $6.9 \mathrm{~g} / \mathrm{dL}$; platelet count, $9.2 \times 10^{4} / \mu \mathrm{L}$; fibrinogen level, 249 $\mathrm{mg} / \mathrm{dL}$; prothrombin time, $12.6 \mathrm{~s}$; and activated partial thromboplastin time, $30 \mathrm{~s}$. Physical examination revealed vaginal laceration and considerable vaginal bleeding on speculum inspection. Because of the inability to stop bleeding, the obstetricians decided to proceed with TAE.

CECT showed a large hematoma near the left vaginal wall and contrast extravasation from the superior rectal artery (Fig. 1). Angiography of the IMA was first performed

Received: January 15, 2021. Accepted: August 12, 2021. Published online in J-STAGE: January 12, 2022.

doi: 10.22575/interventionalradiology.2021-0002

Correspondence Author: Naoki Kawakami, E-mail: n.kawakami19890503@gmail.com 

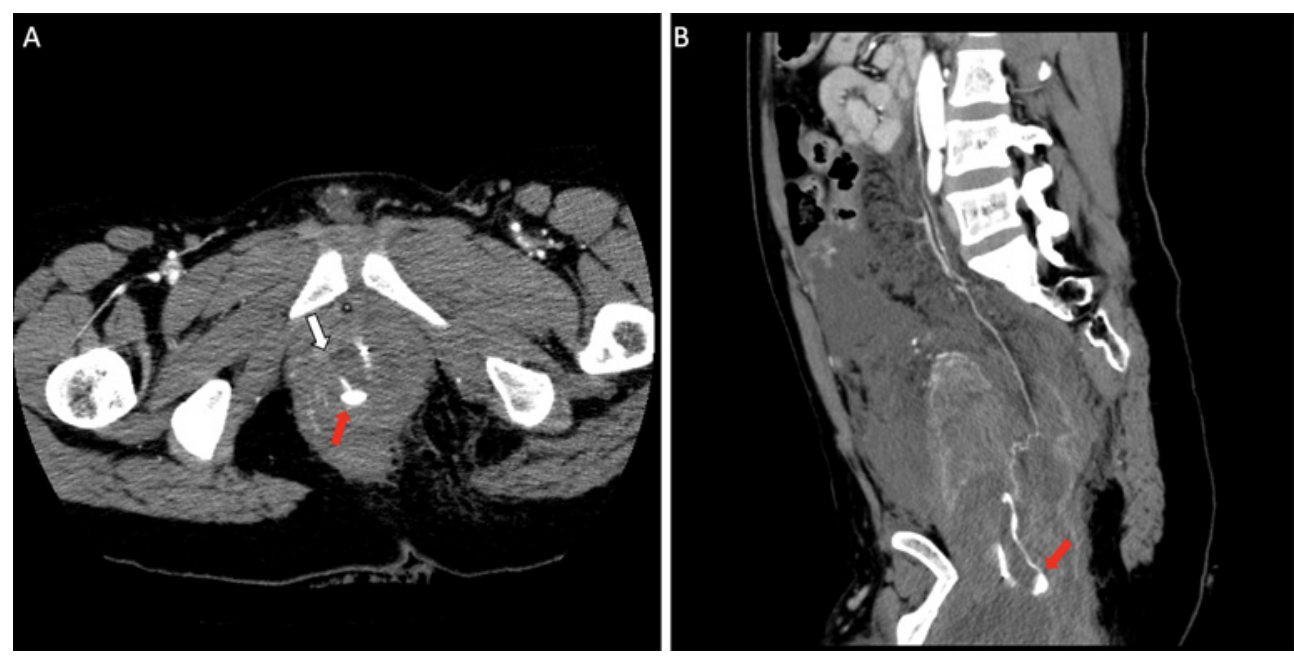

Figure 1. A. Abdominal contrast-enhanced computed tomography (CECT) on admission showing a large hematoma near the left vaginal wall (white arrow) and extravasation from the superior rectal artery (red arrow). B. Curved planar reconstruction (CPR) images showing extravasation from the superior rectal artery (red arrow).
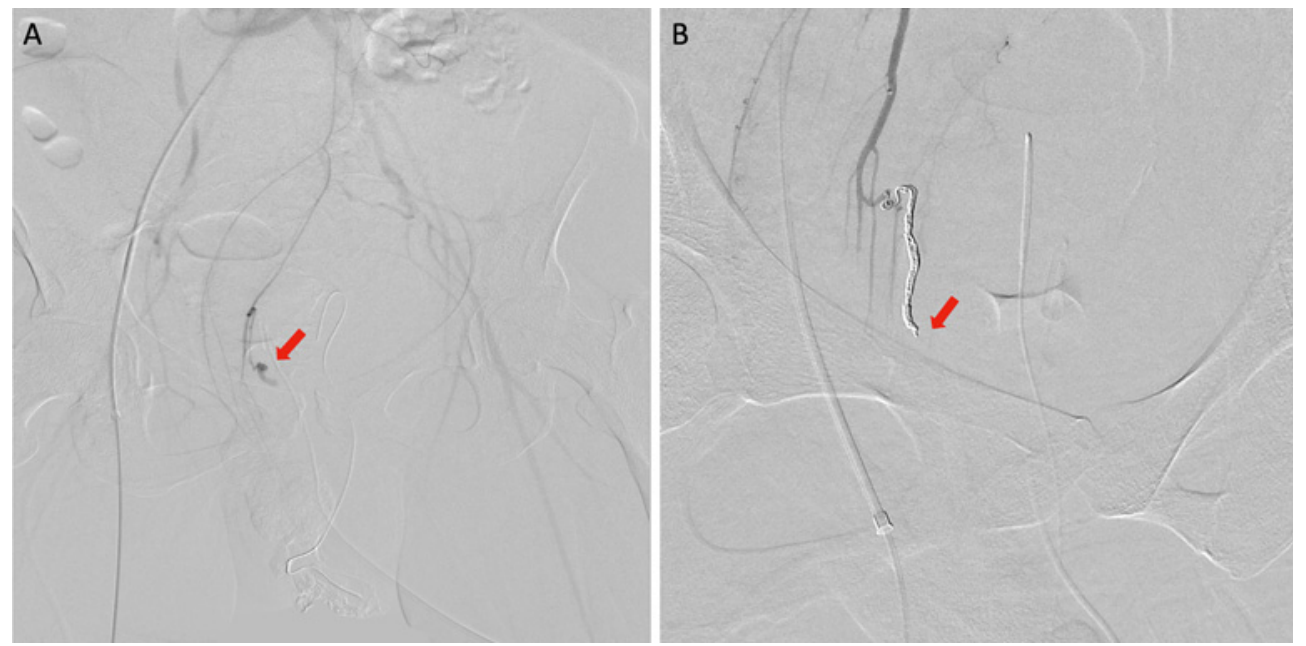

Figure 2. A (RAO $\left.30^{\circ}\right)$. Angiogram of the inferior mesenteric artery (IMA) before transcatheter arterial embolization (TAE) showing extravasation from the superior rectal artery (red arrow). B $\left(A P 0^{\circ}\right.$ ). Successful TAE was performed with five metallic coils: angiogram of the IMA showing no extravasation (red arrow).

via a right femoral artery approach using a 4.2-French catheter (Shepherd hook type; Medikit, Tokyo, Japan). It showed bleeding from a branch of the superior rectal artery (Fig. 2A). Then, a 1.7-French microcatheter (Excelsior SL10, Boston Scientific, Natick, MA, USA) was advanced as close to the hemorrhage site as possible. Embolization was performed using two Target 360 Nano $2 \mathrm{~mm} / 4 \mathrm{~cm}$, one Target 360 Ultra $3 \mathrm{~mm} / 6 \mathrm{~cm}$, and two Target 360 Ultra $3 \mathrm{~mm} / 8$ $\mathrm{cm}$ coils (Stryker, Kalamazoo, MI, USA) (Fig. 2B). Angiography of the bilateral internal iliac arteries was performed using a 4.2-French catheter (Shepherd hook type; Medikit, Tokyo, Japan), which showed no extravasation.

The patient's vital signs and laboratory parameters were stabilized without the need for further intervention. More- over, speculum examination after TAE showed cessation of vaginal bleeding. The patient was discharged after $14 \mathrm{~d}$.

\section{Case 2}

A 27-year-old woman presented with vaginal bleeding that continued for $4 \mathrm{~h}$ after vaginal delivery in our hospital. She had been admitted $8 \mathrm{w}$ before, with a diagnosis of imminent preterm birth. Her vital signs and laboratory data were as follows: blood pressure, $151 / 80 \mathrm{mmHg}$; heart rate, 61 beats/min; shock index, 0.40; hemoglobin level, $10.7 \mathrm{~g} /$ $\mathrm{dL}$; platelet count, $13.3 \times 10^{4} / \mu \mathrm{L}$; fibrinogen level, $411 \mathrm{mg} /$ $\mathrm{dL}$; prothrombin time, $11.5 \mathrm{~s}$; and activated partial thromboplastin time, $29.1 \mathrm{~s}$. Physical examination revealed vaginal laceration and considerable vaginal bleeding on speculum 

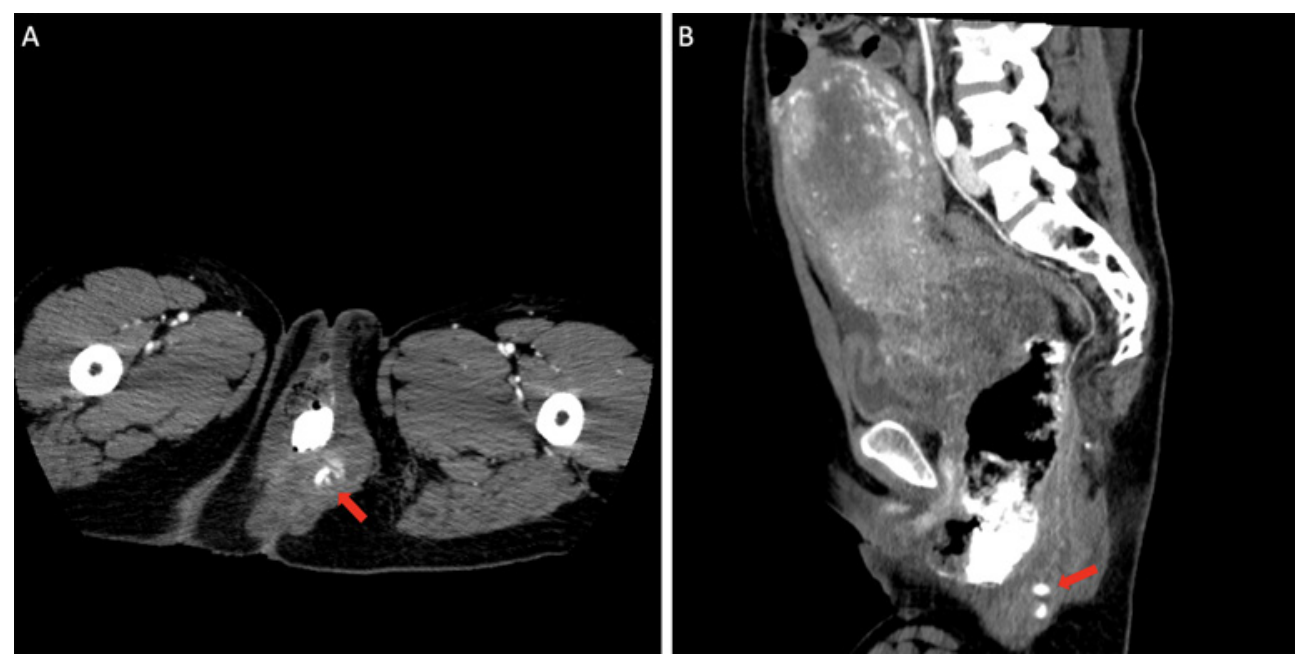

Figure 3. A. Abdominal CECT on the day of delivery showing extravasation from the superior rectal artery (red arrow). B. CPR images showing extravasation from the superior rectal artery (red arrow).
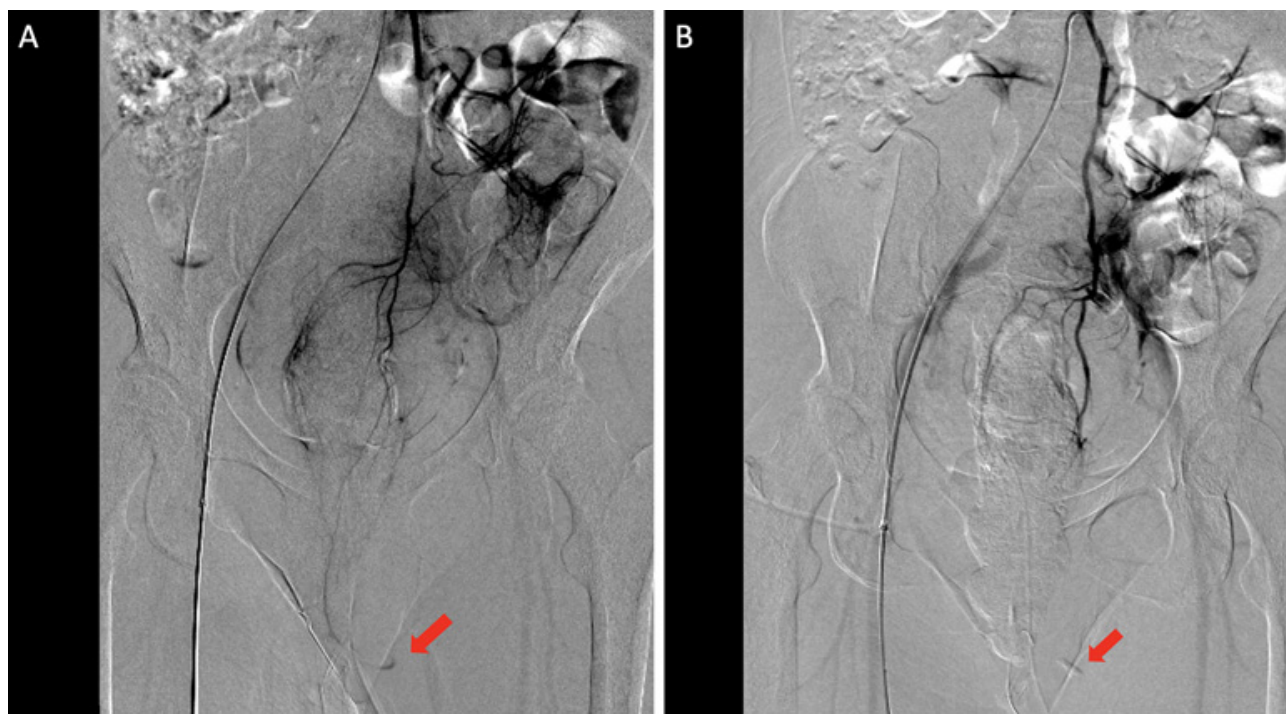

Figure 4. A $\left(\mathrm{AP}^{\circ}\right)$. Angiogram of the IMA showing extravasation from the superior rectal artery (red arrow). B $\left(\mathrm{AP}^{\circ}\right)$. Angiogram of the IMA after TAE with autologous thrombus showing no extravasation (red arrow).

inspection. TAE was selected as the first-line treatment.

CECT showed a large left perineal hematoma and contrast extravasation from the superior rectal artery (Fig. 3). Angiography of the IMA was first performed via a right femoral artery approach using a 5.0-French catheter (Shepherd hook type; Medikit, Tokyo, Japan). It showed bleeding from a branch of the superior rectal artery (Fig. 4A). Then, a 2.0French microcatheter (Excelsior 1,018, Boston Scientific, Natick, MA, USA) was advanced as close to the hemorrhage site as possible. TAE was performed with an autologous thrombus (Fig. 4B). Angiography of the bilateral internal iliac arteries was performed using a 5.0-French catheter (Shepherd hook type; Medikit, Tokyo, Japan), which showed no extravasation.

The patient's vital signs and laboratory parameters were stabilized without the need for repeated interventions. Moreover, speculum inspection confirmed the cessation of bleeding. The patient was discharged after $7 \mathrm{~d}$.

Written informed consent was obtained from both patients for the publication of this report and the accompanying images.

\section{Discussion}

In patients with $\mathrm{PPH}$ due to vaginal laceration, as in our case, the branches of the internal iliac arteries are first assumed to be the source of bleeding. The IMA is a very uncommon bleeding source of PPH [6]. The most probable mechanism for superior rectal artery bleeding is injury to the artery associated with birth canal expansion and lacera- 
tion and extravasation of blood through the torn lower uterine body, cervix, or vagina [4]. Another possibility is direct communication between the distal IMA and uterine artery as an anatomical variant; this unusual collateralization has been reported in patients with prior uterine artery ligation or embolization [7]. The IMA should be considered a source of bleeding in PPH.

Lee et al. reported that CECT could be sensitive in the detection of extravasation [8]. It provides a clue about the culprit artery based on the anatomical location of the hematoma and extravasation. Moreover, the localization of a bleeding artery on CECT can be used as a road map for TAE [8]. We first performed TAE of the IMA on the basis of CECT findings, which led to prompt hemostasis in both cases. When pre-procedural CECT can be performed depending on the patient's general condition, it might help detect the culprit artery and achieve prompt hemostasis.

Various materials, including gelatin sponge particles (GSPs), material coils, and n-butyl cyanoacrylate (NBCA), are used in IMA embolization [6]. GSP is regarded as the embolic agent of choice for PPH. Material coils are used for the proximal occlusion of large vessel lacerations causing rapid extravasation or to avoid extensive nontargeted embolization or occlusion of the pseudoaneurysm. The use of NBCA is considered in arterial rupture, pseudoaneurysm on angiography, and severe coagulopathy [9]. We used a material coil for safe TAE to avoid extensive rectal embolization in Case 1. In Case 2, we accidentally found an autologous thrombus in a syringe during examination and used it, considering it to be suitable for prompt and safe TAE.

In conclusion, the rapid detection of the bleeding artery leads to the effective treatment of patients with PPH. CECT is useful in obtaining clues about the culprit artery based on the anatomical location of the hematoma and extravasation. These useful findings of CECT allow prompt TAE of a rare source of bleeding in PPH, such as the IMA.
Acknowledgement: The authors have no grants and disclosure to report.

\section{Conflict of Interest: None}

\section{References}

1. Woodhams R. The role of interventional radiology in primary postpartum hemorrhage. Hypertens Res Pregnancy 2016; 4: 53-64.

2. Lédée N, Ville Y, Musset D, Mercier F, Frydman R, Fernandez H. Management in intractable obstetric haemorrhage: an audit study on 61 cases. Eur J Obstet Gynecol Reprod Biol 2001; 94: 189196.

3. Chen C, Lee SM, Kim JW, Shin JH. Recent update of embolization of postpartum hemorrhage. Korean J Radiol. 2018; 19: 585596.

4. Kim CH, Jeon GS, Lee SJ, Kang SH. Embolization of the inferior mesenteric artery for post-partum hemorrhage with a vaginal laceration: a case with unusual collateral supply. J Obstet Gynaecol Res 2016; 42: 1878-1880.

5. Shin SM, Yi KW, Chung HH. Postpartum uterine bleeding from the inferior mesenteric artery: case report and review of the literature. Gynecol Obstet Investig 2015; 80: 60-63.

6. Chen C, Chu HH, Shin JH, et al. Inferior mesenteric artery embolization for persistent postpartum hemorrhage after sufficient bilateral iliac arteries embolization: safety and efficacy in eight patients. Br J Radiol 2019; 92: 20180896.

7. Dixon S, Tapping CR, Chuah PS, Bratby M, Uberoi R, Anthony S. Successful fibroid embolization of pelvic and inferior mesenteric artery collaterals after previous uterine artery embolization. Acta Radiol 2012; 53: 292-295.

8. Lee SM, Shin JH, Shim JJ, et al. Postpartum haemorrhage due to genital tract injury after vaginal delivery: safety and efficacy of transcatheter arterial embolization. Eur Radiol 2018; 28: 48004809.

9. Lee NK, Kim S, Kim CW, Lee JW, Jeon UB, Suh DS. Identification of bleeding sites in patients with postpartum hemorrhage: MDCT compared with angiography. AJR 2010; 194: 383-390.

Interventional Radiology is an Open Access journal distributed under the Creative Commons Attribution-NonCommercial 4.0 International License. To view the details of this license, please visit (https://creativecommons.org/licenses/by$\mathrm{nc} / 4.0 /)$. 